\title{
XMM-Newton optical monitor observations of LMC X-3
}

\author{
R. Soria ${ }^{1}, \mathrm{~K} . \mathrm{Wu}^{1,2}$, M. J. Page ${ }^{1}$, and I. Sakelliou ${ }^{1}$ \\ 1 Mullard Space Science Laboratory, University College London, Holmbury St Mary, Dorking, RH5 6NT, UK \\ 2 School of Physics, University of Sydney, Sydney 2006, NSW, Australia
}

Received 2 October 2000 / Accepted 26 October 2000

\begin{abstract}
We study the optical counterpart of the black-hole X-ray binary LMC X-3, by using XMM-Newton/OM observations carried out during a low-hard X-ray state. We derive a better constraint for the temperature, mass and radius of the companion star, and we show that the star is likely to be a $\sim$ B5 subgiant filling its Roche lobe. Taking into account the effect of X-ray irradiation, we suggest a value $f_{\mathrm{M}} \approx 1.5 M_{\odot}$ for the mass function in this system, lower than previously thought; we provide a more accurate lower limit to the mass of the compact object.
\end{abstract}

Key words. accretion, accretion disks - black hole physics - binaries: general - stars: early type - X-rays: general

\section{Introduction}

An important unsolved problem for the black-hole candidate (BHC) LMC $\mathrm{X}-3$ is the process of mass transfer. With an estimated mass of the companion star $4 M_{\odot} \lesssim M_{2} \lesssim 8 M_{\odot}$ (Cowley et al. 1983), the system appears to be intermediate between high-mass black-hole binaries like Cyg X-1 (mass of the companion star $\approx 33 M_{\odot}$, see Giles \& Bolton 1986), and low-mass black-hole binaries like A0620-00 (mass of the companion star $\approx 0.7 M_{\odot}$ ). In the former class of systems, mass transfer occurs mainly via a stellar wind, and the donor star is more massive than the primary; in the latter, the donor star is usually a late-type star filling its Roche lobe.

The optical counterpart (Warren \& Penfold 1975) of LMC X-3 shows ellipsoidal modulations with a total amplitude of $\lesssim 0.2 \mathrm{mag}$ and a period corresponding to the 1.705 d binary period (van der Klis et al. 1983; van der Klis et al. 1985). It also shows long-term brightness variations in the range $16.7 \lesssim V \lesssim 17.5$ (e.g., van Paradijs et al. 1987). Its optical spectrum was found to be consistent with that of a B3 main sequence star (Cowley et al. 1983). From the radial velocity shifts of the optical absorption lines, Cowley et al. (1983) derived a mass function $f_{\mathrm{M}}=2.3 M_{\odot}$, thus establishing this system as a strong BHC. However, the spectral identification of the companion star remains uncertain (Mazeh et al. 1986) because of the effect of X-ray irradiation on the secondary, and the possible contribution to the optical flux by an accretion disk. In fact, the long-term variations in both the optical

Send offprint requests to: R. Soria,

e-mail: rs1@mssl.ucl.ac.uk colours and the $V$ brightness are found to be associated with changes in the soft X-ray flux (Cowley et al. 1991).

Moreover, Nowak et al. (2000) and Wilms et al. (2000) suggest that spectral transitions between a high-soft and a low-hard state could be associated with changes in the accretion rate. A more correct identification of the companion star can provide a better understanding of the physics of mass transfer and state transitions.

\section{XMM-Newton optical monitor observations}

LMC X-3 was observed with the Optical Monitor 30 in telescope (Mason et al. 1996) on board XMM-Newton on 2000 April 19 (MJD 51653; XMM-Newton revolution 66). A $\log$ of the observations is reported in Table 1. The system was in a low-hard state during our optical observation, with a pure power-law X-ray spectrum with photon index $\approx 1.6-1.8$ (Boyd \& Smale 2000; Wu et al. 2001). We estimate from simultaneous $X M M-N e w t o n / P N$ observations (Wu et al. 2001) that the $2-10 \mathrm{keV}$ flux was $\lesssim 10^{36} \mathrm{erg} \mathrm{s}^{-1}$, at least two orders of magnitude lower than in the high-soft state (Nowak et al. 2000; Wilms et al. 2000). This is in agreement with the RXTE/ASM 2-10 keV lightcurve. Therefore, optical photometric observations of the companion star at this epoch provide the best chance to determine its intrinsic colours and spectral type.

\section{Optical colours and temperature}

The average brightness of LMC $\mathrm{X}-3$ measured in the three XMM-Newton/OM optical bands on 2000 April 19 
was $v=17.48 \pm 0.02, b=17.39 \pm 0.02, u=16.56 \pm$ 0.02. (For a detailed comparison between the $X M M$ Newton/OM photometric system and Johnson's standard $U B V$ system, see Royer et al. 2000). Using the latest available matrix of colour transformation coefficients (Version 4, 2000 September), we find that this corresponds to $V=17.47 \pm 0.03, B=17.35 \pm 0.03, U=16.77 \pm 0.03$.

Our observations covered a fraction of the binary phase; therefore they may not yield the true phaseaveraged brightness of the optical counterpart. If we adopt the ephemeris of van der Klis (1985), the $V$ images were centred at phase $\phi=0.86 \pm 0.10$, the $B$ images at $\phi=0.95 \pm 0.10$ and the $U$ images at $\phi=0.90 \pm 0.10$. However, those ephemeris may have accumulated too large an error to be still reliable. From the amplitude of the ellipsoidal variations, we estimate that there is an additional error of $0.08 \mathrm{mag}$ in the $V$ brightness, of $0.03 \mathrm{mag}$ in the $U-B$ colour, and of 0.04 mag in the $B-V$ colour.

If we assume a reddening $E(B-V)=0.06 \pm 0.01$ (Schlegel et al. 1998; Crampton 1979) and a distance modulus $d=18.5 \pm 0.1$, we obtain intrinsic colours $(B-V)_{0}=$ $-0.17 \pm 0.05,(U-B)_{0}=-0.64 \pm 0.04$, and an absolute magnitude $M_{V}=-1.22 \pm 0.16$ for the optical counterpart of $\mathrm{LMC} \mathrm{X}-3$.

Both optical colour indices can be used as indicators of the effective temperature $T_{\text {eff }}$. From Buser \& Kurucz (1978), and Cramer (1984), we find that these intrinsic colours correspond to stars with $15500 \lesssim T_{\text {eff }} \lesssim 17000 \mathrm{~K}$. The colour-temperature relation is almost independent of surface gravity, so that it can be applied to both main sequence and giant stars. We also checked the effect of different metal abundances by using the semi-empirical colour-temperature calibration of Lejeune et al. (1998). For $[\mathrm{Fe} / \mathrm{H}]=-0.3$, our observed colour indices imply $15600 \lesssim T_{\text {eff }} \lesssim 17200$, while for $[\mathrm{Fe} / \mathrm{H}]=-1.5$ they imply $16300 \lesssim T_{\text {eff }} \lesssim 18000$. If we adopt the slightly bluer colours for the companion star measured by van der Klis et al. (1983), we obtain temperatures $T_{\text {eff }} \approx 18000-19000$.

From the catalogue of Lejeune et al. (1998) we derive the bolometric corrections, which depend on the temperature and (weakly) on the metal abundance, but are almost independent of surface gravity. From the inferred range of bolometric luminosities and temperatures we obtain a reliable constraint to the mass of the secondary star.

Figure 1 shows the single-star evolutionary tracks from Girardi et al. (2000) in the $\left(\log T_{\text {eff }}, \log L_{\text {bol }}\right)$ plane, for a metal abundance $Z=0.008$ (a typical value for the LMC, see for example Caputo et al. 1999). Assuming that evolutionary tracks for stars in binary systems can be approximated by single-star tracks, we infer that the constraint is satisfied by evolved stars with $4.7 M_{\odot} \lesssim M_{2} \lesssim 5.3 M_{\odot}$ corresponding to subgiants of spectral type $\sim \mathrm{B} 5$. A B3 main sequence companion $\left(M_{2} \approx 6-7 M_{\odot}\right)$ has the correct luminosity but much higher temperatures. This result is not very sensitive to the adopted value of metal abundance.

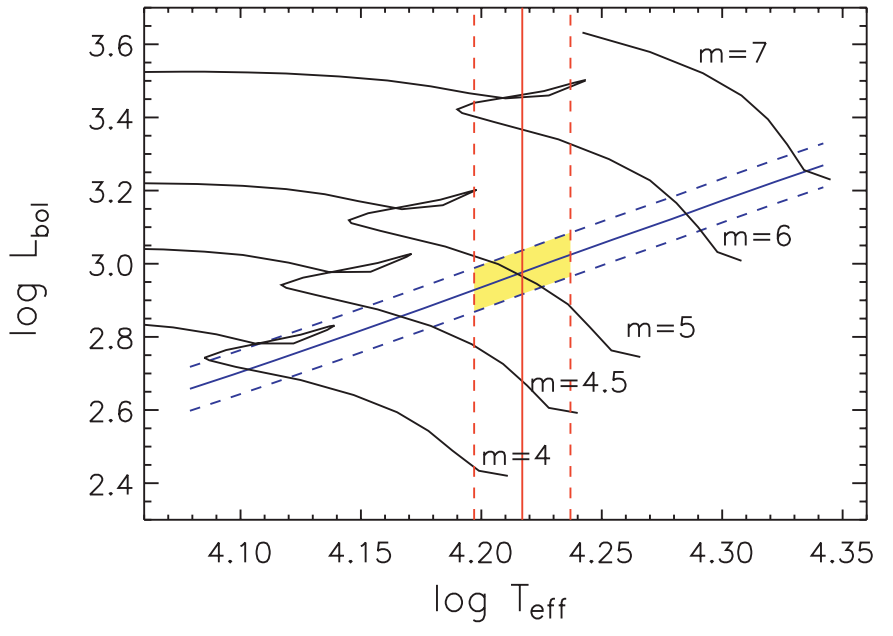

Fig. 1. Evolutionary tracks for stars of various masses, at $Z=0.008$, in the $\left(\log T_{\text {eff }}, \log L_{\text {bol }}\right)$ plane, with the acceptable range of temperatures and luminosities derived from our $X M M-N e w t o n / O M$ observations. Masses are in units of solar mass, $M_{\odot}=1.9910^{33} \mathrm{~g}$; temperature is in $\mathrm{K}$; luminosity is in units of solar bolometric luminosity, $L_{\mathrm{bol}, \odot}=3.910^{33} \mathrm{erg} \mathrm{s}^{-1}$

Table 1. Log of our XMM-Newton/OM observations

\begin{tabular}{ccc}
\hline OM filter & Mid-exposure time (MJD) & Exposure time (s) \\
\hline$v$ & 51653.148 & 1000 \\
$v$ & 51653.178 & 1000 \\
$v$ & 51653.193 & 1000 \\
$v$ & 51653.208 & 1000 \\
$u$ & 51653.123 & 1000 \\
$u$ & 51653.238 & 1000 \\
$u$ & 51653.253 & 1000 \\
$u$ & 51653.268 & 1000 \\
$u$ & 51653.283 & 1000 \\
$b$ & 51653.298 & 1000 \\
$b$ & 51653.313 & 1000 \\
$b$ & 51653.328 & 1000 \\
$b$ & 51653.343 & 1000 \\
$b$ & 51653.358 & 1000 \\
\hline
\end{tabular}

\section{Radius of the companion star}

The mean mass density in the Roche lobe of the companion star is uniquely determined by the orbital period $P$ (Frank et al. 1992):

$\rho \equiv \frac{3 M_{2}}{4 \pi R_{\mathrm{L}}^{3}} \approx 115 P_{\mathrm{hr}}^{-2} \approx 0.069 \mathrm{~g} \mathrm{~cm}^{-3}$,

where $R_{\mathrm{L}}$ is the mean radius of the Roche lobe. We plot in Fig. 2 the evolutionary tracks (Girardi et al. 2000) in the $\left(M_{V}, \rho\right)$ plane, together with the mean density in the Roche lobe derived in Eq. (1), for $Z=0.008$. The dashed line corresponds to a radius of $0.95 R_{\mathrm{L}}$. Stars with $4 M_{\odot} \lesssim M_{2} \lesssim 4.7 M_{\odot}$ would be very close to filling their Roche lobe at the observed brightness $M_{V}$. If the companion star has a mass $M_{2}=4.7 M_{\odot}$, its radius would be $R_{2}=4.4 R_{\odot} \simeq R_{\mathrm{L}}$. Mass transfer would mainly occur via 




Fig. 2. Evolutionary tracks for stars of various masses, at $Z=$ 0.008 , in the $\left(M_{V}, \rho\right)$ plane, compared with the absolute $V$ brightness derived from our XMM-Newton/OM observations, and the mean density inside the Roche lobe of the secondary star. The dashed line corresponds to a radius $R=0.95 R_{\mathrm{L}}$ for which Roche-lobe overflow becomes significant

Roche-lobe overflow. Stars of lower mass would also fill their Roche lobe but are ruled out by the observed range of colours (Fig. 1). On the other hand, if $M_{2}>4.7 M_{\odot}$, the companion star would not fill its Roche lobe. In particular, a B3V companion $\left(R_{2} \approx 4 R_{\odot}\right)$ would only fill less than half of the volume of its Roche lobe. The main mechanism of mass transfer would have to be a stellar wind. If the wind were to account for X-ray luminosities up to $\approx 410^{38} \mathrm{erg} \mathrm{s}^{-1}$ in the high-soft state (Johnston et al. 1979), the column density would be much higher than $10^{21} \mathrm{~cm}^{-2}$, difficult to reconcile with the result obtained from the XMM-Newton X-ray observations (Wu et al. 2001).

The evolutionary time-scale for a subgiant star of mass $\approx 4.7 M_{\odot}$ in the observed range of temperature is $\approx 210^{7}$ years (Girardi et al. 2000). If the mass transfer is driven by the nuclear evolution of the secondary, this would imply a mass-transfer rate $\approx 210^{-7} M_{\odot}$ per year, sufficient to account for luminosities $\gtrsim 10^{39} \mathrm{erg} \mathrm{s}^{-1}$.

\section{A lower limit on the mass of the primary}

The masses of the two components of a binary system are given by

$M_{\mathrm{X}}=\frac{(1+q)^{2}}{\sin ^{3} i} f_{\mathrm{M}}$,

$M_{2}=\frac{q(1+q)^{2}}{\sin ^{3} i} f_{\mathrm{M}}$

where $q \equiv M_{2} / M_{\mathrm{X}}$ and $f_{\mathrm{M}}$ is the mass function of the primary, which can be obtained from spectroscopic observations. The X-rays are not eclipsed (Cowley et al. 1983); if the secondary star is approximated by a sphere, this implies (Paczyński 1983):

$R_{2}<K_{2} \frac{P}{2 \pi}(1+q) \cot i$, where $K_{2}$ is the projected radial velocity semi-amplitude of the secondary star. From Eqs. (3) and (4), an upper limit for $q$ is obtained:

$$
\begin{aligned}
& R_{2}< K_{2} P(2 \pi)^{-1}(1+q)^{1 / 3} q^{-1 / 3} f_{\mathrm{M}}^{-1 / 3} M_{2}^{1 / 3} \\
& \times\left[1-q^{2 / 3}(1+q)^{4 / 3} f_{\mathrm{M}}^{2 / 3} M_{2}^{-2 / 3}\right]^{1 / 2},
\end{aligned}
$$

where the right-hand side is a monotonically decreasing function of $q$ where defined.

From the radial velocity shifts of the stellar absorption lines, Cowley et al. (1983) determined $K_{2}=235 \pm$ $11 \mathrm{~km} \mathrm{~s}^{-1}$ and $f_{\mathrm{M}}=2.3 \pm 0.3 M_{\odot}$. Using these values together with the values of $M_{2}$ and $R_{2}$ determined in Sect. 4, we obtain from Eq. (5):

$M_{\mathrm{X}}>7.3 \pm 0.6 M_{\odot}$.

As an aside, we also note that if we assume a lower limit $i>50$ for the inclination angle (van der Klis et al. 1985), Eq. (2) gives an upper limit $M_{\mathrm{X}}<13.8 \pm 1.0 M_{\odot}$.

If the secondary star is really a B5 subgiant, its intrinsic luminosity is $\lesssim 3.510^{36} \mathrm{erg} \mathrm{s}^{-1}$ (Fig. 1), corresponding to an intrinsic flux $F_{2} \lesssim 310^{12} \mathrm{erg} \mathrm{cm}^{-2} \mathrm{~s}^{-1}$. The radial velocity curve of the stellar absorption lines was obtained by Cowley et al. (1983) when the source was in a high state, implying an X-ray luminosity $L_{\mathrm{X}} \gtrsim 10^{38} \mathrm{erg} \mathrm{s}^{-1}$. The orbital separation in a binary system is $a=2.910^{11} M_{1}^{1 / 3}(1+q)^{1 / 3} P_{\mathrm{d}}^{2 / 3} \approx$ $1.010^{12} \mathrm{~cm}$ for LMC $\mathrm{X}-3$. Hence, the energy intercepted by the secondary star per unit time is $(1 / 4) L_{\mathrm{X}}\left(R_{2} / a^{2}\right)^{2} \gtrsim 310^{36} \mathrm{erg} \mathrm{s}^{-1}$, comparable with the intrinsic luminosity of the star.

In a spherical approximations, the intercepted flux is larger than the intrinsic flux on $\approx 1 / 4$ of the surface of the star, for a soft X-ray luminosity $\approx 210^{38} \mathrm{erg} \mathrm{s}^{-1}$, and for a disk with $H / R \lesssim 0.2$. Strong external irradiation with soft X-rays tends to cause photo-ionisation at the surface of the secondary star. As a consequence, absorption lines from the irradiated face should be weakened or suppressed, and the observed radial velocity shifts may not reflect the true orbital motion of the centre of mass of the secondary (Wade \& Horne 1988; Phillips et al. 1999).

Using the model previously applied to the BHC GRO J1655-40 (Phillips et al. 1999), and assuming for simplicity that no absorption lines are produced in the region where the external flux is larger than the internal flux, we can estimate the effect of irradiation in the case of LMC $\mathrm{X}-3$. The observed amplitude of the radial velocity variations of the absorption lines $\left(K_{2}\right)$ should appear larger than the true radial velocity amplitude of the centre of mass of the secondary $\left(K_{2}^{\prime} \equiv K_{2}-\Delta K_{2}\right)$. For the parameters of LMC X-3, we estimate that the correction is $\Delta K_{2}=30 \pm 5 \mathrm{~km} \mathrm{~s}^{-1}$. Therefore, the true $K$-velocity of the secondary star is only

$K_{2}^{\prime}=205 \pm 12 \mathrm{~km} \mathrm{~s}^{-1}$.

Hence, after the correction, the mass function $\left(\propto K_{2}^{3}\right)$ for the compact object in LMC $\mathrm{X}-3$ is

$f_{\mathrm{M}}=1.5 \pm 0.3 M_{\odot}$. 
By using these revised $K$-velocity and mass function in Eq. (5), we obtain a lower limit to the mass of the compact object:

$M_{\mathrm{X}}>5.8 \pm 0.6 M_{\odot}$.

It would be important to test this prediction by determining the radial velocity curve of the stellar lines during a low X-ray state. We also note that if the irradiated side of the secondary star contributes only to the optical continuum flux but not to the absorption lines, their relative strength would appear reduced in comparison with a nonirradiated star of the same spectral type. This is a possible explanation of what was observed by Cowley et al. (1983).

\section{Conclusions}

We have observed the BHC LMC X-3 with the Optical Monitor on board XMM-Newton during an X-ray low-hard state. The $2-10 \mathrm{keV}$ flux at that epoch was the lowest ever observed by RXTE/ASM, consistent with zero. The brightness and colours of the optical counterpart inferred from our observations are likely to be a good approximation of the intrinsic values of the companion star. This allows us to constrain the mass and radius of the secondary. We have found that an evolved subgiant of mass $M_{2} \approx 4.7 M_{\odot}$ and temperature $T_{\text {eff }} \approx 16500 \mathrm{~K}$ (spectral type $\sim \mathrm{B} 5 \mathrm{IV}$ ) is consistent with the observed colours and luminosity. We have also shown that such a star would be filling its Roche lobe, thus explaining why the source is mostly observed in a high-soft state, dominated by an accretion disk. No significant wind is expected from such star, in agreement with the low column density inferred from the XMM-Newton/EPIC and RGS X-ray data. The evolutionary time-scale of a B5 IV star is also consistent with the observed X-ray luminosity powered by nuclearevolution driven mass transfer.

The companion star was previously thought to be a main-sequence B3 star. Although it would have the same brightness, a B3 V star would fill only about one half of its Roche lobe, ruling out mass transfer via Roche-lobe overflow.

A B5 IV companion implies a lower limit to the mass of the primary $M_{\mathrm{X}}>7.3 \pm 0.6 M_{\odot}$, if we assume the $K$-velocity and mass function determined by Cowley et al. (1983). However, the spectroscopic observations of Cowley et al. (1983) were carried out during an X-ray active state, when the effect of soft X-ray irradiation on the surface of the secondary star is significant. The true radial velocity amplitude of the centre of mass of the secondary is probably $\approx 12-15$ per cent less than the value observed by Cowley et al. (1983). This would reduce the mass function to $f_{\mathrm{M}}=1.5 \pm 0.3 M_{\odot}$, and set a lower limit $M_{\mathrm{X}}>5.8 \pm 0.6 M_{\odot}$ for the mass of the compact object.

Acknowledgements. KW acknowledges the support from the ARC Australian Research Fellowship and a PPARC visiting fellowship. We thank Keith Mason for his comments.

\section{References}

Boyd, P. T., \& Smale, A. P. 2000, IAUC, 7424

Buser, R., \& Kurucz, R. L. 1978, A\&A, 70, 555

Caputo, M., Marconi, G., \& Ripepi, V. 1999, ApJ, 525, 784

Cowley, A. P., Crampton, D., Hutchings, J. B., Remillard, R., \& Penfold, J. E. 1983, ApJ, 272, 118

Cowley, A. P., Schmidtke, P. C., Ebisawa, K., et al. 1991, ApJ, 381,526

Cramer, N. 1984, A\&A, 132, 283

Crampton, D. 1979, ApJ, 230, 717

Frank, J., King, A., \& Raine, D. 1992, Accretion Power in Astrophysics (Cambridge: University Press)

Giles, D. R., \& Bolton, C. T. 1986, ApJ, 304, 37

Girardi, L., Bressan, A., Bertelli, G., \& Chiosi, C. 2000, A\&AS, 141,371

Johnston, M. D., Bradt, H. V., \& Doxsey, R. E. 1979, ApJ, 233, 514

Lejeune, T., Cuisinier, F., \& Buser, R. 1998, A\&AS, 130, 65

Mason, K. O., Cropper, M. S., Hunt, R., et al. 1996, Proc. SPIE 2808, 438, ed. O. H. Siegmund, \& M. A. Gummin

Mazeh, T., van Paradijs, J., van den Heuvel, E. P. J., \& Savonije, G. J. 1986, A\&A, 157, 113

Nowak, M. A., Wilms, J., Heindl, W. A., et al. 2000, MNRAS, in press [astro-ph/0005487]

Paczyński, B. 1983, ApJ, 273, L81

Phillips, S. N., Shahbaz, T., \& Podsiadlowski, Ph. 1999, MNRAS, 304, 839

Royer, P., Manfroid, J., Gosset, E., \& Vreux, J.-M. 2000, A\&AS, 145, 351

Schlegel, D. J., Finkbeiner, D. P., \& Davis, M. 1998, ApJ, 500, 525

van der Klis, M., Tjemkes, S., \& van Paradijs, J. 1983, A\&A, 126, 265 (see also Erratum 132, 240 (1984))

van der Klis, M., Clausen, J. V., Jensen, K., Tjemkes, S., \&van Paradijs, J. 1985, A\&A, 151, 322

van Paradijs, J., van der Klis, M., Augusteijn, T., et al. 1987, A\&A, 184, 201

Wade, R. A., \& Horne, K. 1988, ApJ, 324, 411

Warren, P. R., \& Penfold, J. E. 1975, MNRAS, 172, 41P

Wilms, J., Nowak, M. A., Pottschmidt, K., et al. 2000, MNRAS, in press [astro-ph/0005489]

Wu, K., Soria, R., Page, M. J., et al. 2001, A\&A, 365, L267 